\title{
Research Square \\ Capital Expenditure and Operating Efficiency from Vertical Integration in the Global Semiconductor Industry
}

Guangshun Qiao ( $\nabla$ qiaoguangshun@wzbc.edu.cn )

Wenzhou Business College, Wenzhou

Research

Keywords: nonparametric conditional efficiency, directional distance estimator, capital expenditure, global semiconductor industry, integrated device manufacturer

Posted Date: September 15th, 2021

DOI: https://doi.org/10.21203/rs.3.rs-871733/v1

License: (c) (1) This work is licensed under a Creative Commons Attribution 4.0 International License.

Read Full License 


\title{
Capital Expenditure and Operating Efficiency from Vertical Integration in the Global Semiconductor Industry
}

\author{
Guangshun Qiao*
}

September 2021

\begin{abstract}
This paper uses a nonparametric production frontier approach to investigate the operating efficiency differences by the impacts of capital expenditure and business model in the global semiconductor industry. Handling the impact of capital expenditure as fixed input by the directional distance estimator, this study compares the operating efficiencies in the global semiconductor industry between the integrated device manufacturers and the fabless and foundry firms over 1999-2018. The estimation results indicate that vertically integrated manufacturers dominate the semiconductor industry, and the capital-intensive companies operate more efficiently than the asset-light fabless firms on average.
\end{abstract}

Keywords: nonparametric conditional efficiency, directional distance estimator, capital expenditure, global semiconductor industry, integrated device manufacturer

*School of Finance and Trade, Wenzhou Business College, Wenzhou, 325035, China; email qiaoguangshun@wzbc.edu.cn. 


\section{Introduction}

Semiconductors, also referred to as chips or integrated circuits (ICs), are essential components of virtually all modern electronic devices. Since Bell laboratories invented the transistors in 1947 and the first commercial ICs released in the 1960s, the semiconductor industry has been at the forefront of the growth of the digital economy. Prior to the 1980s, integrated device manufacturers (IDMs), which internally perform all of the production processes (e.g., research and design (RD), front-end fabrication, and back-end assembly and test (AT)), dominated the semiconductor industry. As semiconductors with ever-expanding complexity approach the limits of Moore's Law (e.g., see Mack, 2011 and Flamm, 2017), the expenses of manufacturing an inventive chip have become prohibitive for almost all the IC suppliers. These increasing costs stimulated business model innovation and gave birth to the fabless-foundry business model in the mid-1980s. In this new business model, fabless companies dedicate their time to IC design and brand operation. At the same time, pure-play foundries devote themselves to front-end fabrication, and a third group of companies are allotted for back-end outsourced semiconductor assembly and test (OSAT) operations. The fabless-foundry business model has significantly changed the structure of the semiconductor industry over the last few decades.

The structural changes by specialization in the semiconductor value chain is a topic of wide interest (e.g., see Macher et al., 2007, Adner and Kapoor, 2010 and Sarma and Sun, 2017). Although the semiconductor industry is both technology-intensive and capital-intensive, much research on the topic of the structural change in the semiconductor industry emphasizes the impact of evolving technologies (e.g., see Macher, 2006, Kapoor and Adner, 2012 and Hwang and Choung, 2014), while the impact of capital investments has not been discussed adequately. Simultaneously, vertical disintegration in the semiconductor value chain is accompanied and twisted by the trend of industry globalization (e.g., see Brown et al., 2005), which further increases the complexity of the topic. Recent developments in nonparametric frontier estimation (e.g., see Daraio 
and Simar, 2014 and Daraio et al., 2020) provide tools to analyze the operating efficiencies in the semiconductor industry under various types of constraints such as capital investments and the business model. This paper aims to use the Daraio et al. (2020) approach to shed light on disentangling the impact of capital investments and comparing the technical efficiencies between the IDMs and the vertical disintegrated fabless and foundry firms in the semiconductor industry. Data on 470 companies in North America, Europe, and Asia are collected to provide a worldwide perspective of the highly globalized semiconductor industry.

Before jumping into the details of the nonparametric frontier estimation, it is worth tracing the origin and evolution of the fabless-foundry business model in the semiconductor industry. A milestone of the vertical disintegration in the semiconductor industry is the establishment of the Taiwan Semiconductor Manufacturing Company (TSMC) in 1987. Committed to be a long-term non-competitive partner with fabless firms, TSMC is the first and nowadays the largest pure-play foundry worldwide that dedicates to wafer fabrication (e.g., see Hsieh et al., 2002). The drastically reduced burden of capital expenditure (CAPEX) and the reduced barriers to entry by vertical specialization ensure domination of new markets by the fabless firms. The collaboration between the asset-light fabless and the pure-play foundry also provides the more robust protection of intellectual property (IP) rights when the fabless firms pass on their design blueprints to pure-play foundries, which earlier are exposed to the threats of replication and IP theft when fabless firms' ICs are manufactured by their rival IDMs (e.g., see Sarma and Sun, 2017). The entry of new fabless companies, most of which are spinoffs from industry incumbents, spur innovation and propel the diversification of products in various applications. Since the 1990s, fabless firms have had substantial shares or even dominated in most of the fastest-growing market segments (e.g., see Balconi and Fontana, 2011). Nevertheless, despite a trend toward vertical specialization driven by the entry of fabless firms, the vertical integrated IDMs have continued to persist and coexist with the fabless entrants in the semiconductor industry. 
Factors that determine production costs and operating efficiencies vary essentially across device types and business models in the capital-intensive and technology-intensive semiconductor industry. On the one hand, manufacturing is capital intensive for leadingedge products such as microprocessors and radiofrequency devices, with steadily rising R\&D and equipment expenditures. The slow progress in node technology requires continuous investments in both $\mathrm{R} \& \mathrm{D}$ and advanced fabrication facilities. The uncertainties set high barriers to entry and favor the success of large IDMs, such as Intel, STMicroelectronics, and Texas Instruments, which are able to make risky investments and have a higher chance to foresee and lead the technology evolutions. On the other hand, the major challenges for front-end fabrication and back-end A\&T procedures are the heavy CAPEX for cleanroom and costly equipment. The foundries and OSATs seek to optimize productivity by serving many fabless companies to achieve high capacity utilization. In comparison, the fabless companies, many of which are niche startups, getting rid of the burden in setting up, maintaining, and upgrading fabrication facilities, focus on R\&D to compete with the IDMs. Hence CAPEX is a crucial factor in the semiconductor industry with a feature of lump sum fixed input.

There has been a long-lasting debate on which business model is operating more efficiently or is more likely to dominate the semiconductor industry. On one side, Monteverde (1995) and Dibiaggio (2007) credit the efficiency of IDMs to the internalization of transaction costs. Ernst (2005), Macher (2006) and Kapoor and Adner (2012) hold the knowledge-based view that the IDMs achieve performance advantages when technological developments involve complex problems. On the other side, Li et al. (2011) show that foundries are becoming technology transferors rather than merely manufacturing capacity providers in the semiconductor industry value chain. Kapoor (2013) proposes and finds that the incumbents who persist with vertical integration increase their emphasis on systemic innovations. Besides the examples shown above that focus on analyzing the impact of technology evolution in the semiconductor industry, this paper plans to emphasize the feature of capital intensive in the semiconductor indus- 
try and focus on exploring the impacts of CAPEX and business model on operating efficiency.

Taking advantage of a flexible functional form, data envelopment analysis (DEA) is one of the most popular approaches for efficiency estimation. There are rich records for performance evaluation in the semiconductor industry using the DEA approach. For instance, Kozmetsky and Yue (1998) examine the cost efficiency of 56 IC companies worldwide and show that US, Japanese, South Korean, and Taiwanese IC companies have become the major participants in the global semiconductor industry in the early 1990s. Lu and Hung (2010) compare the managerial performance efficiency of 48 leading vertically disintegrated firms in Taiwan's IC value chain and note that fabless companies perform better than foundries and OSATs. Jang et al. (2016) measure the cumulative change in R\&D efficiency of 49 global leading fabless companies and note that during the period 2007-2013, the overall R\&D efficiency declines slightly. Li et al. (2019) explore 64 major Chinese enterprises in the semiconductor industry and find that low levels of scale efficiency is the most significant factor limiting future improvements to innovation efficiency. One common problem of these studies, among others, such as Lu et al. (2013), Hung et al. (2014), Hsu (2015) and Tsai et al. (2017), is the slow convergence rate of the nonparametric DEA estimator accompany by the increasing numbers of input and output dimensions.

The issue of slow convergence rate in DEA estimation may become severe if the observations are restricted to a small number either by geographic boundary or by business model boundary. For example, the researches of Wu et al. (2006), Lu et al. (2010), and Kuo and Yang (2012) use a small number of 38-39 companies to evaluate the performance of the fabless corporations in Taiwan, while in some extreme cases, such as Hung and Lu (2008), Liu and Wang (2008), Chen and Chen (2011), and Lin et al. (2019), the studies contain only 10-25 companies, which may lead to unconvincing results. It also explains why the Free Disposal Hull (FDH) estimator, which is known as an unbiased substitution of the DEA estimator but with a much slower convergence 
rate, is not widely used in empirical works (e.g., see Wilson, 2018). One solution to mitigate the slow convergence rate problem in nonparametric efficiency estimation is to increase the number of observations by considering the deeply globalized semiconductor industry as an aggregated market. This paper follows this strategy to investigate the impacts of the business model and CAPEX in the highly globalized semiconductor industry through a conditional nonparametric frontier approach. The heterogeneity by CAPEX is treated as a fixed input variable by the directional distance estimator (e.g., see Daraio et al., 2020), while the heterogeneity by the business model is handled by the conditional efficiency estimators (e.g., see Daraio and Simar, 2007). The estimation results indicate that vertically integrated manufacturers dominate the semiconductor industry and the capital-intensive IDMs and OSATs operate more efficiently than the asset-light fabless firms on average.

The paper is organized as follows. Section 2 gives the nonparametric frontier framework and discusses the diagnostics and test statistics for choosing a suitable estimator in this research. Section 3 presents the empirical results and discusses the effect of capital investment and business model in the semiconductor industry. The last section concludes.

\section{Methods}

\subsection{The Nonparametric Frontier Model}

The economic theory of efficiency in production can be traced to the ideas of Koopmans (1951), Debreu (1951) and Farrel (1957). Consider a production process in which $p$ inputs are used to produce $q$ outputs. The production set

$$
\Psi=\left\{(x, y) \in \mathbb{R}_{+}^{p+q} \mid x \text { can produce } y\right\}
$$

describes the set of attainable combinations of inputs and outputs. The efficiency score of a particular production plan $(x, y)$ is then determined by the distance from $(x, y)$ to the efficient frontier or boundary of $\Psi$ (denoted as $\Psi^{\partial}$ ). There are four kinds of 
commonly used efficiency measures based on different directions in which the distance is calculated. The most widely used radial measures are the input- and output-oriented Debreu-Farrel measures. Färe et al. (1985) introduce the hyperbolic measure

$$
\gamma(x, y \mid \Psi)=\inf \left\{\gamma \mid\left(\gamma x, \gamma^{-1} y\right) \in \Psi\right\}
$$

as an alternative to selecting either an input- or output-oriented Debreu-Farrel measures, where input and output quantities are adjusted simultaneously to reach the boundary $\Psi^{\partial}$ along a hyperbolic path. Note that these three kinds of efficiency measures are all radial measures that allow for only nonnegative values of inputs and outputs.

Chambers et al. (1998) propose an additive measure of the technical efficiency, known as the directional distance measure, which considers adding the feasible quantities to a unit's output and simultaneously subtracting proportional quantities from its input. In detail, the directional distance measure is given by

$$
\beta\left(x, y \mid d_{x}, d_{y}, \Psi\right)=\sup \left\{\beta \mid\left(x-\beta d_{x}, y+\beta d_{y}\right) \in \Psi\right\}
$$

which projects the input-output vector $(x, y)$ onto the technology in a specified direction $\left(-d_{x}, d_{y}\right)$ and allows for negative values of $x$ and $y$. Note that the directional distance measure $\beta\left(x, y \mid d_{x}, d_{y}, \Psi\right)$ nests both input- and output-oriented DebreuFarrel measures as special cases by setting the direction vector $\left(d_{x}, d_{y}\right)$ as $(x, 0)$ and $(0, y)$, respectively. The flexibility of the directional distance measure also comes from the choices of the directions $d_{x}$ and $d_{y}$ that some directions (but not all) can be set equal to zero to represent non-discretionary inputs or outputs (e.g., see Simar and Vanhems, 2012). This feature of the directional distance measure can represent the impact of a kind of fixed input or output variables, such as CAPEX, which can be categorized into input variables of production but are not under managers' direct control, at least in the short run. 
The attainable set $\Psi$ is unobserved in empirical works. Nonparametric methods such as FDH and DEA are developed to estimate the unobservable production set $\Psi$. Deprin et al. (1984) define the FDH estimator $\widehat{\Psi}_{\mathrm{FDH}}$ as

$$
\widehat{\Psi}_{\mathrm{FDH}}=\bigcup_{X_{i}, Y_{i} \in \mathcal{S}_{n}}\left\{(x, y) \in \mathbb{R}_{+}^{p+q} \mid x \geq X_{i}, y \leq Y_{i}\right\}
$$

where $\mathcal{S}_{n}=\left\{\left(X_{i}, Y_{i}\right)\right\}$ denote a random sample of $n$ pairs of inputs and outputs. FDH estimators of $\widehat{\gamma}_{\mathrm{FDH}}(x, y \mid \Psi)$ and $\widehat{\beta}_{\mathrm{FDH}}\left(x, y \mid d_{x}, d_{y}, \Psi\right)$ are obtained by replacing $\Psi$ with $\widehat{\Psi}_{\text {FDH }}$ in (2.2)-(2.3) respectively. Baker et al. (1984) propose the varing-returns-to-scale DEA (VRS-DEA) estimator $\widehat{\Psi}_{\text {VRS }}$ as the convex hull of $\widehat{\Psi}_{\mathrm{FDH}}$. The corresponding VRSDEA estimators of $\widehat{\gamma}_{\mathrm{VRS}}(x, y \mid \Psi)$ and $\widehat{\beta}_{\mathrm{VRS}}\left(x, y \mid d_{x}, d_{y}, \Psi\right)$ are obtained by replacing $\Psi$ with $\widehat{\Psi}_{\text {VRS }}$ in (2.2)-(2.3) respectively. There is another kind of the DEA estimator, defined as the convex cone of $\widehat{\Psi}_{\mathrm{FDH}}$ and called the constant-returns-to-scale DEA (CRSDEA) estimator. Since the CRS-DEA estimator relies on strong assumption that the returns to scale are everywhere constant, it is less widely used in empirical works. Therefore, the notation DEA is dedicated for VRS-DEA for the rest of the paper.

Besides inputs and outputs, there exist factors such as differences in ownership, business models, constraints of technology and regulatory, which are typically beyond control of the manager but may influence the production process. These factors (denoted as environmental factors $Z \in \mathbb{R}^{r}$,) may not be independent of $(X, Y)$, so that the unknown effect of $Z$ must be estimated appropriately. Daraio and Simar (2005) propose a framework to investigate the joint behavior of $(X, Y, Z)$ in probability terms by defining the conditional attainable set as

$$
\Psi^{z}=\left\{(x, y) \in \mathbb{R}_{+}^{p+q} \mid x \text { can produce } y \text { when } Z=z\right\}
$$

where $\Psi=\bigcup_{z \in Z} \Psi^{z}$, so that $\Psi^{z} \subseteq \Psi$, for all $z \in Z$. Then the distribution of $(X, Y)$ 
conditional on $Z=z$ is denoted by

$$
H_{X, Y \mid Z}(x, y \mid z)=\operatorname{Prob}(X \leq x, Y \geq y \mid Z=z)
$$

which gives the probability that a firm facing environmental conditions $z$ will dominate the point $(x, y)$. Given $Z=z$, the attainable set $\Psi^{z}$ is the support of $H_{X, Y \mid Z}(x, y \mid z)$.

Introducing environmental factors into (2.2)-(2.3) extend the efficiency scores into their conditional counterparts. For example, the conditional hypobolic measure can be expressed as

$$
\gamma(x, y \mid z)=\inf \left\{\gamma \mid H_{X, Y \mid Z}\left(\gamma x, \gamma^{-1} y \mid z\right)>0\right\}
$$

and the conditional directional distance measure can be expressed as

$$
\beta\left(x, y \mid d_{x}, d_{y}, z\right)=\sup \left\{\beta \mid H_{X, Y \mid Z}\left(x-\beta d_{x}, y+\beta d_{y} \mid z\right)>0\right\} .
$$

Therefore, plugging a nonparametric estimator of $H_{X, Y \mid Z}(\cdot)$ from a sample $S_{n}=$ $\left\{X_{i}, Y_{i}, Z_{i} \mid i=1, \ldots, n\right\}$ into (2.7) or (2.8) can derive the estimation of the conditional efficiency scores accordingly. Such a nonparametric estimator of $H_{X, Y \mid Z}(\cdot)$ may be obtained by standard kernel smoothing, for example,

$$
\widehat{H}_{X, Y \mid Z}(x, y \mid z)=\frac{\sum_{i}^{n} \boldsymbol{I}\left(X_{i} \leq x, Y_{i} \geq y\right) K\left(\frac{Z_{i}-z}{h}\right)}{\sum_{i}^{n} K\left(\frac{Z_{i}-z}{h}\right)},
$$

where $K(\cdot)$ is a kernel function with bounded support, $h$ is a vector of bandwidths $h=\left(h_{1}, \ldots, h_{r}\right)$, and $r$ is the number of environmental variables. It is well known that the selection of bandwidth $h$ is of critical importance in kernal smoothing (e.g., see Hall et al., 2004, Bădin et al., 2010, 2012, Jeong et al., 2010, and Li et al., 2013 for the discussions of choosing the optimal bandwidth by least square cross-validation $(\mathrm{LSCV}))$.

There is a particular case, called the separability condition in Simar and Wilson (2007), where $Z$ has no impact on the boundaries of the $\Psi^{z}$ and $\Psi^{z}=\Psi$ for all $z \in Z$. 
Simar and Wilson $(2007,2011)$ emphasize that if the separability condition is not hold, naive regression in a second-stage analysis may provide inconsistent estimation. Alternatively, Bădin et al. (2012, 2014) suggest a flexible nonparametric location-scale model

$$
\gamma(X, Y \mid Z=z)=\mu(z)+\sigma(z) \varepsilon
$$

in a second-stage regression, where $\mu(z)$ measures the average effect of $z$ on the efficiency, and $\sigma(z)$ provides additional information on the dispersion of the efficiency distribution as a function of $z$. Mastromarco and Simar (2015), Cordero et al. (2017), Toma (2020), etc., had used this two-step approach for various empirical studies.

\subsection{Estimation and Inference}

The tradeoff between FDH and DEA estimators for performance evaluation is not trival. Simar and Wilson (2015) summarize in a survey of the nonparametric frontier models that the FDH and DEA estimators converge to limiting distributions at rates $n^{\frac{1}{p+q}}$ and $n^{\frac{2}{p+q+1}}$ respectively under appropriate assumptions. Hence for a fixed sample size $n$, the convergence rate slows down with the increasing of dimensionality $(p+q)$, which increases the estimation error accordingly for both the FDH and DEA estimators. A feasible approach to minimize such estimation error (often referred to as the curse of dimensionality) is either to increase the sample size $n$ or to decrease the total dimensions of $(p+q)$. If the sample size $n$ is restricted to a small number by real world constraints, including the market scale and market scope in specific industries, geographical or political restrictions, and the high cost of data collection, dimension reduction may become an attractive solution.

Daraio and Simar (2007, pp. 148-150) propose using principal component analysis (PCA) for dimension reduction. Wilson (2018) explains how to use PCA for a mapping $\Psi: R_{+}^{p+q} \longmapsto R_{+}^{1+1}$ for the radial measures. In detail, $(p \times n)$ matrix $\boldsymbol{X}$ and $(q \times n)$ matrix $\boldsymbol{Y}$ are transformed to $(1 \times n)$ matrices $\Lambda_{x_{1}}^{\prime} \boldsymbol{X}, \Lambda_{y_{1}}^{\prime} \boldsymbol{Y}$ by pre-multiplying the first eigenvector $\Lambda_{x_{1}}, \Lambda_{y_{1}}$ of the moment matrices $\boldsymbol{X} \boldsymbol{X}^{\prime}$ and $\boldsymbol{Y} \boldsymbol{Y}^{\prime}$. Though it is not 
possible to give a theorem that precisely identifies situations where dimension reduction should be used, Wilson (2018) provides three diagnostics for empirical research. The first diagnostic is to compute the effective parametric sample size $m$ as $m \approx\left\lfloor n^{2 \kappa}\right\rceil$, where $\kappa=\frac{1}{p+q}$ for FDH estimator, $\kappa=\frac{2}{p+q+1}$ for DEA estimator, and $\lfloor a\rceil$ denotes the integer nearest $a$. Hence the criterion of judging the minimum sample size $m$ in parametric estimation can be used as reference in judging the minumum sample size $n$ in nonparametric estimation.

A second diagnostic is to consider the proportion of $n$ observations that yield efficiency scores equal to one. Since FDH estimator converges slower than DEA estimator, a robust diagnostic for the curse of dimensionality should use the FDH efficiency estimator. If more than $25 \%-50 \%$ of the observations yield efficiency scores equal to one, the estimation results are not convincing. A third diagnostic is to exam the ratios $R_{x}$ and $R_{y}$ of the largest eigenvalue of the moment matrices $\boldsymbol{X} \boldsymbol{X}^{\prime}$ and $\boldsymbol{Y} \boldsymbol{Y}^{\prime}$ to the corresponding sum of eigenvalues for $\boldsymbol{X} \boldsymbol{X}^{\prime}$ and $\boldsymbol{Y} \boldsymbol{Y}^{\prime}$, which provide measures of how close the corresponding moment matrices are to rank-one. For example, if $R_{x}=0.9$, then the matrix with dimension reduction $\Lambda_{x 1}^{\prime} \boldsymbol{X}$ contains $90 \%$ of the independent linear information in the original matrix $\boldsymbol{X}$. In practice, Wilson (2018) proposes standardizing the matrices $\boldsymbol{X}$ or $\boldsymbol{Y}$ before PCA to ensure the inputs or outputs have the same scale, in case of excessive number of inputs or outputs.

After the diagnostics of dimension reduction, Kneip et al. (2015, 2016) use new central limit theorems to construct a test of convexity for the tradeoff between $\widehat{\Psi}_{\mathrm{FDH}}$ and $\widehat{\Psi}_{\text {VRS }}$. Note that the main difference of the test statistics by the new central limit theorems in Kneip et al. $(2015,2016)$ are the bias corrections construced by jackknife estimators (e.g., see Kneip et al., 2016, pp. 441-442). If the null hypothesis of convexity is rejected, the FDH estimator is the only consistent estimator. Alternatively, if the null hypothesis of convexity is not rejected, though it does not imply that the null is true, the DEA estimator may be the preferred estimator because of its faster convergence rate. However, the test of convexity proposed by Kneip et al. (2015, 2016) depends on 
randomly split the original sample into two independent subsamples for the calculation of the bias terms, which introduces ambiguity in practice. Simar and Wilson (2020, pp. 293-294) develop a generalized bootstrap algorithm that eliminates much of this ambiguity by repeating the random splits a large number of times, which can be used for both the convexity test and the separability test.

\subsection{Data and Variable Specification}

The data are collected from the Sub-Industry of Semiconductors in the Compustat database. In order to provide a global perspective for the semiconductor value chain, I combine data from both the Compustat North America database and the Compustat Global database to cover companies in the industry worldwide. As the semiconductor industry is famous for being a cyclical industry (e.g., see Tan and Mathews, 2010), I gather twenty years of data between 1999-2018 to cover a sufficient period with multiple business cycles in the industry. The reason for the data to begin in 1999 is twofold. First, with ten years of development since the inception of the fabless-foundry business model in the late-1980s, the global semiconductor value chain has been preliminarily established in the late-1990s so that there are plenty of available annual reports for the fabless and foundry firms on the open market and in Compustat database. Second, two years after the 1997 Asian financial crisis, the year 1999 is a suitable starting point to observe the trend in the global semiconductor industry without massive exogenous shocks for the following years until the 2008 financial crisis. I also exclude liquid crystal display manufacturers, light-emitting diodes, manufacturers and photovoltaic producers from the dataset, limiting the sample to only IC manufacturers in a narrow sense. Hence the panel data include 5136 observations from 470 unique companies in the global semiconductor industry in 1999-2018.

A side product of the flexible functional form in the nonparametric frontier approach is a lack of theoretical foundation on the production function. Identifying the inputs and outputs has always been a subject of controversy, either in parametric or nonparametric frontier estimations, without exception in the semiconductor industry. 
Hence I sort the most commonly used variables in 37 empirical papers which apply the nonparametric frontier approach for performance evaluation in the semiconductor industry. Besides a few variables which are chosen for specific topics, the commonly used variables in these papers are highly concentrated into two input categories and two output categories. The first input category measures all kinds of variable inputs, including labor, raw material, R\&D and sales, and marketing expenditure, while the second input category measures fixed assets. Comparably, the first output category measures revenue and the second output category measures the market value of the firms. Therefore, I specify $p=5$ inputs (labor, measured by the number of employees $\left(X_{1}\right)$; COGS $\left(X_{2}\right)$; R\&D expenditure $\left(X_{3}\right)$; sales and marketing expenditure $\left(X_{4}\right)$ and fixed assets, measured by property, plant, and equipment (PP\&E) $\left.\left(X_{f}\right)\right)$ and $q=2$ outputs (total revenue $\left(Y_{1}\right)$; and shareholders' equity, measured by common ordinary equity (CEQ) $\left.\left(Y_{2}\right)\right)$. Since I plan to use the directional distance estimator, I distinguish the notation of the fixed input $X_{f}$ with the other four variable inputs $X_{1}, X_{2}, X_{3}$, and $X_{4}$. For the output variable $Y_{2}$, I use shareholders' equity instead of the market value of a firm, because the variable of market value is suffering from missing data in the Compustat database, and the variable shareholders' equity is also a widely used proxy for the value of a firm.

Table 1 gives summary statistics for the original variables in 1999-2018 pooled data. In order to provide a uniform standard across years, all the variables except $X_{1}$ are expressed in millions of U.S. dollars and their values have been adjusted to 2018 U.S. dollar by GDP deflator. The distribution of all the variables are heavily skewed to the right, owning to the domination of several semiconductor giants in the market. In addition, I specify $r=2$ environmental variables (business model $\left(Z_{1}\right)$; and time, measured by the years $\left.1999-2018\left(Z_{2}\right)\right)$. The environmental variable $Z_{1}$ is a discrete variable, which categorize the four kinds of business models including fabless, IDM, foundry, and A\&T into three groups. The first group contains fabless companies which are labor intensive for chip design, while the second group contains both foundries and 
OSATs which are capital intensive for fabrication, and a third group contains IDMs which are both labor intensive and capital intensive. The environmental variable $Z_{2}$ can either be treated as a continuous variable or a discrete variable, which will be discussed further in the next section.

Table 2 breaks down the 5136 observations by business model. It is no surprise that over half of the companies are fabless. As the barriers to entry, which relies heavily on CAPEX, is much lower for fabless than for the others, fabless companies spring up like the mushrooms in the late-1990s to the early-2000s. At the same time, the number of firms operating in other kinds of business models remain relatively stable. After the golden decade of fast growth in the semiconductor industry come to an end in the mid-2000s (e.g., see Flamm 2017), the proportions of firms in each business model are gradually fixed. Around $60 \%$ of the firms are fabless, while $20 \%$ of the firms are IDMs and the rest $20 \%$ are either front-end wafer fabs or back-end OSATs.

\section{Results and Discussion}

It is well known that most nonparametric estimators suffer from the curse of dimensionality. Based on the three diagnostics introduced above, the necessity for dimension reduction is unambiguous. With seven dimensions $(p=5$ and $q=2)$ in the original data, the effective parametric sample size $m$ for the original annual data is small, no matter using FDH or DEA estimators. A slight difference in processing PCA for the directional distance estimator is that PCA is only on the variable inputs and outputs, but not on the fixed input $X_{f}$. Hence after dimension reduction there remain three dimensions including $\widetilde{X}$ (PCA from $X_{1}$ to $X_{4}$ ), $\widetilde{Y}$ (PCA from $Y_{1}$ to $Y_{2}$ ) and $X_{f}$. I calculate the values of the largest eigenvalue of the moment matrices of $\boldsymbol{X} \boldsymbol{X}^{\prime}$ and $\boldsymbol{Y} \boldsymbol{Y}^{\prime}$ to the corresponding sum of eigenvalues to be $R_{x}=91.19 \%$ and $R_{y}=98.31 \%$, indicating high correlations among the input variables $X_{1}, \ldots, X_{4}$ and high correlations between the output variables $Y_{1}, \ldots, Y_{2}$, so that dimension reduction should reduce estimation error. Thus all of the following analyses and results are based on data with dimension reduciton. 
Among studies that use nonparametric frontier approach to estimate efficiency and benchmark performance of firms in the semiconductor industry, the vast majority choose DEA estimator, without comparing the pros and cons between FDH estimator and DEA estimator. The DEA estimator is probably a better choice without dimension reduction, as the slower convergence rate of FDH estimator may increase measurement error rapidly with the increasing of dimensions. However, it is worth to reevaluate the tradeoff between FDH estimator and DEA estimator with dimension reduction. The drawback of DEA estimator is imposing convexity on the production set $\Psi$, while FDH estimator is free of this assumption. As discussed above, the test of convexity versus non-convexity of the product set proposed by Kneip et al. (2016) can be applied to measure this tradeoff. The FEAR package (e.g., see Wilson, 2008) uses a bootstrap algorithm by Simar and Wilson (2020) to extend the Kneip et al. (2016) approach for the convexity test. Table 3 provides results of the convexity test, using the FEAR package and the choosing hyperbolic-oriented measure in (2.2). At 95\% confidence level, the null hypothesis of convexity are rejected for over $80 \%$ of the 20 years annual data, except 3 years (2009, 2011 and 2012) in hyperbolic-orientation. Simar and Vanhems (2012) link the directional distance measure with the standard hyperbolic measure by a monotonic transformation, so that the results in Table 3 are also valid for the directional distance estimator. Hence I choose FDH estimator for the remainder of the analysis.

Daraio et al. (2020) propose a fast and efficient computation of the directional distance measures using FDH estimator. In detail, after monotonic transformation of the data that

$$
X^{*}=\tilde{X} \oslash d_{x} \quad \text { and } \quad Y^{*}=\tilde{Y} \oslash d_{y}
$$

where $\oslash$ refers to Hadamard component-wise division of vectors, the FDH estimator 
in (2.4) can be expressed explicitly as

$$
\begin{aligned}
\widehat{\beta}\left(x, y \mid d_{x}, d_{y}\right) & =\sup \left\{\beta>0 \mid \widehat{H}_{n, X^{*} Y^{*} \mid X_{f}}\left(x^{*}-\beta, y^{*}+\beta \mid x_{f}\right)>0\right\}, \\
& =\max _{\left\{i \mid X_{f, i} \leq x_{f}\right\}}\left[\min \left\{x^{*}-X_{i}^{*}, Y_{i}^{*}-y^{*}\right\}\right]
\end{aligned}
$$

where $n$ is the sample size and $i \in\{1,2, \ldots, n\}$. It is straightforward to extend the expression in (3.2) to the conditional directional distance estimator (e.g., see Daraio et al., 2020, pp. 814) as

$$
\widehat{\beta}\left(x, y \mid d_{x}, d_{y}, z\right)=\max _{\left\{i\left|X_{f, i} \leq x_{f},\right| Z_{i}-z \mid \leq h\right\}}\left[\min \left\{x^{*}-X_{i}^{*}, Y_{i}^{*}-y^{*}\right\}\right]
$$

Therefore, in order to consider a discrete environmental variable such as the business model $Z_{1}$ for the estimator in (3.3), the separability condition needs to be examined. Similarly, in order to consider a continuous environmental variable such as $Z_{2}$ (in case $Z_{2}$ is treated as continuous) for the estimator in (3.3), the optimal bandwidth $h$ needs to be fixed in advance.

Simar and Wilson (2020) propose a bootstrap algorithm which can be used for the separability test on the discrete environmental variable $Z_{1}$. In application to the additive directional distance measure, step [5] in Simar and Wilson (2020, pp. 293) which is originally designed for the radial measures should be revised as

$$
X_{i}^{*}=X_{i}-\widehat{\beta}_{i} \times d_{x}+\beta_{i}^{*} \times d_{x}
$$

and

$$
Y_{i}^{*}=Y_{i}+\widehat{\beta}_{i} \times d_{y}-\beta_{i}^{*} \times d_{y}
$$

where the directions of $d_{x}$ and $d_{y}$ are chosen commonly as the sample mean of $X$ and $Y$. The first portion in Table 4 shows the separability test results with respect to the business model $Z_{1}$. Though the test statistics $\tau_{1}$ and $\tau_{2}$ not always give the same results, there is strong evidence to reject the separability condition. In other words, 
each of the three business models in semiconductor industry has its unique production frontier for pooled data.

For the environmental variable $Z_{2}$ which represents the years 1999-2018, there is flexibility to either treat it as a discrete variable or as a continuous variable (e.g., see Mastromarco and Simar, 2015). To treat $Z_{2}$ as a discrete variable, the 20 years of 1999-2018 can be splitted into ten 2-year groups (two adjacent years as a group), five 4-year groups (four adjacent years as a group) or four 5-year groups (five adjacent years as a group). In this case the separability test with respect to $Z_{2}$ is similar to the separability test with respect to $Z_{1}$. Although $Z_{2}$ can naturally be treated as 20 individual years, it is not recommended for the directional distance measure here. Since each individual year has around 100-300 observations, the effective parametric sample size $m=n^{\frac{2}{3}}$ for the directional distance measure in each year will be a small number and hence increase the measurement error and make this approach less attrative.

Another approach is to treat $Z_{2}$ as a continuous variable and using LSCV to determine the optimal bandwidth $h$ by minimizing

$$
\frac{\sum_{i=1}^{n} \sum_{j \neq i}^{n}\left[\mathrm{I}\left(\widetilde{x}_{i} \leq \widetilde{x}_{j}, x_{f, i} \leq x_{f, j}, \widetilde{y}_{i} \geq \widetilde{y}_{j}\right)-\frac{\frac{1}{n} \sum_{k \neq i}^{n} \mathrm{I}\left(\widetilde{x}_{k} \leq \widetilde{x}_{j}, x_{f, k} \leq x_{f, j}, \widetilde{y}_{k} \geq \widetilde{y}_{j}\right) K_{h}\left(z_{i}, z_{k}\right)}{\frac{1}{n-1} \sum_{k \neq i}^{n} K_{h}\left(z_{i}, z_{k}\right)}\right]^{2}}{n(n-1)} .
$$

The optimal bandwidth is $h=5.5$ for fabless, IDM, and pooled data, implying the smoothing window of year $t$ is $[\mathrm{t}-5, \mathrm{t}+5]$, while the optimal bandwidth is $h=7.5$ for OSAT with the smoothing window of year $t$ to be $[t-7, t+7]$. The second portion in Table 4 shows the separability test results with respect to the optimal time $Z_{2}$, while the third portion in Table 4 shows the separability test results with respect to both the business model $Z_{1}$ and the time $Z_{2}$. In any case the separability conditions are strongly rejected. Hence the efficiency scores in (3.3) are estimated with separated production frontiers per the restriction of both the conditions $Z_{1}$ and $Z_{2}$.

Table 5 shows the summary of the efficiency scores conditional on both the business 
model $Z_{1}$ and time $Z_{2}$. Whether the time $Z_{2}$ is treated as a discrete variable or a continuous variable, the distributions of the efficiency scores are skewed to the right in all kinds of business models, especially for the fabless firms. Nevertheless, on conditions that $Z_{2}$ are treated as a discrete variable, the first quartiles are either equal to zero or very close to zero, no matter how the years are grouped. Based on the second diagnostic in Wilson (2018), it is a sign that the measurement error by slow convergence rate still exist. Choosing a estimation method with larger subsample size is a feasible solution to minimize such measurement error with dimension reduction. Thus a preferred approach is to treat $Z_{2}$ as a continuous variable, rewarding faster convergence rate and more accurate estimates.

Figure 1 visualizes the trends of the annual mean efficiencies by business model. Either treating $Z_{2}$ as a discrete variable or as a continuous variable, the curves of the annual mean efficiencies for the fabless firms are above the curves for the other business models. This phenomenon is more visable in the bottom right panel, where $Z_{2}$ is defined as a continuous variable with more reliable estimates. As higher efficiency score infers lower technical efficiency in the directional distance measure, the curves in Figure 1 imply the fabless firms are operating less efficiently on average. Another interesting discovery is that the curves of different business models in the bottom right panel of Figure 1 tend to converge in 2008, the year of global finanical crisis. It can be interpreted that under extreme conditions the differences in operating efficiency become unconspicuous among business models. Based on the bottom right panel of Figure 1 which produce more accurate estimates, I use new central limit theorem (e.g., see Kneip et al., 2015, pp. 409) to derive $95 \%$ confidence interval for the annual mean efficiency curves in Figure 2. The variance for the fabless firms are also higher comparing with the IDMs or OSATs, implying higher risk and uncertainty for the fabless business model.

As the separability condition does not hold, I use a flexible nonparametric locationscale model in (2.10) for a second-stage regression. The pure efficiency defined by Bădin 
et al. (2012) can be derived from (2.10) and expressed as

$$
\widehat{\varepsilon}(z)=\frac{\widehat{\beta}(x, y \mid z)-\widehat{\mu}(z)}{\widehat{\sigma}(z)} .
$$

In practice, I obtain $\widehat{\mu}(z)$ by regressing $\widehat{\beta}(x, y \mid z)$ on the environmental variable $z$ and $\widehat{\sigma}(z)$ by regressing the squared residuals of the preceding regression on $z$. The upper panel in Figure 3 illustrates the pure efficiency $\widehat{\varepsilon}\left(z_{1}, z_{2}\right)$ that cleanses efficiency scores from the influence of both the environmental factors $Z_{1}$ and $Z_{2}$, while the lower panel in Figure 3 illustrates the pure efficiency $\widehat{\varepsilon}\left(z_{2}\right)$ that cleanses efficiency scores from the influence of only the environmental factor $Z_{2}$. In the upper panel, the curves of $\widehat{\varepsilon}\left(z_{1}, z_{2}\right)$ by different business models twist together with no clear structures, similar to white noise vibrating at small values around zero. On the contrary, in the lower panel, the curves of $\widehat{\varepsilon}\left(z_{2}\right)$ demonstrate clear separation by business models. Since $\widehat{\varepsilon}\left(z_{2}\right)$ only cleanses the influence of time, the lower panel in Figure 3 maintains the structure of the differences in technical efficiency by the business model in Figure 1-2. Consequently, the contrast between the upper and lower panels in Figure 3 provides further evidence that the technical efficiencies do vary in the semiconductor by business models, and the asset-light fabless firms are operating less efficiently on average in the past two decades.

\section{Conclusion}

The semiconductor industry is famous for the high barriers to entry, especially in the capital-intensive manufacturing portion. The incumbent IDMs, benefitted by the economy of scale and protected by the economic moat by huge CAPEX, dominate the semiconductor industry since the onset of the industry. However, wagering on novel technologies and processes with the ever-expanding complexity of ICs becomes a weighty burden even for the giant IDMs. The fabless-foundry business model alleviates the financial risks of capital investment, reduces the barriers to entry, accelerats technology iterations, and leads to a flourishing of fabless design houses for various 
applications. This paper compares the operating efficiencies between the IDMs and the fabless-foundry business models to shed light on which business model will be the market trend and dominate the semiconductor industry in the long run.

Based on the capital-intensive feature of the semiconductor industry, this paper chooses a directional distance measure to handle the constraint of CAPEX. At the same time, conditional FDH estimators are used to handle the effects of business model and time in the nonparametric frontier approach. The empirical results provide clear evidence that the fabless firms are operating less efficiently on average. Though the fabless-foundry business model encourages entrance of the fabless startups, the CAPEX barriers accompanying with technical barriers still limit the fields and applications for the fabless firms to growth and development. The IDMs have more room to optimize the operation and lead the technology development with a strategic product roadmap by vertical integration. The fabless-foundry business model is more like a complement of the IDMs to explore a broader scope in the semiconductor industry instead of a substitutional structure change. The IDMs will continuously dominate the semiconductor industry in the foreseeable future. 


\section{Declarations}

\section{Ethics approval and consent to participate}

Not applicable

\section{Consent for publication}

Not applicable

\section{Availability of data and materials}

The data used in the current study are available from the corresponding author on request.

\section{Competing interests}

The author declare that he has no competing interests.

\section{Funding}

Not applicable

\section{Author's contributions}

Not applicable

\section{Acknowledgements}

I am particularly grateful to Paul W. Wilson for his advice. 


\section{References}

Adner, R., Kapoor, R., 2010, Value creation in innovation ecosystems: how the structure of technological interdependence affects firm performance in new technology generations, Strategic Management Journal, Vol. 31, pp. 306-333.

Bădin, L., Daraio, C., Simar, L., 2010, Optimal bandwidth selection for conditional efficiency measures: A data-driven approach, European Journal of Operational Research, Vol. 201, pp. 633-640.

Bădin, L., Daraio, C., Simar, L., 2012, How to measure the impact of environmental factors in a nonparametric production model, European Journal of Operational Research, Vol. 223, pp. 818-833.

Bădin, L., Daraio, C., Simar, L., 2014, Explaining inefficiency in nonparametric production models: the state of the art, Annals of Operations Research, Vol. 214, pp. $5-30$.

Balconi, M., Fontana, R., 2011, Entry and innovation: an analysis of the fabless semiconductor business, Small Business Economics, Vol. 37, pp. 87-106.

Banker, R.D., Charnes, A., Cooper, W.W., 1984, Some models for estimating technical and scale inefficiencies in data envelopment analysis, Management Science, Vol. 30, pp. 1078-1092.

Brown, C., Linden, G., Macher, J., 2005, Offshoring in the semiconductor industry: a historical perspective, Brookings Trade Forum, pp. 279-333.

Chambers, R.G., Chung, Y.H., Färe, R., 1998, Profit, directional distance functions, and Nerlovian efficiency, Journal of Optimization Theory and Applications, Vol. 98, pp. 351-364.

Chen, Y.S., Chen, B.Y., 2011, Applying DEA, MPI, and grey model to explore the operation performance of the Taiwanese wafer fabrication industry, Technological Forecasting \& Social Change, Vol. 78, pp. 536-546.

Cordero, J.M., Pedraja-Chaparro, F., Pisaflores, E.C., Polo, C., 2017, Efficiency assessment of Portuguese municipalities using a conditional nonparametric approach, Journal of Productivity Analysis, Vol. 48, pp. 1-24.

Daraio, C., Simar, L., 2005, Introducing environmental variables in nonparametric frontier models: A probabilistic approach, Journal Productivity Analysis, Vol. 24, pp. 93-121.

Daraio, C., Simar, L., 2007, Advanced Robust and Nonparametric Methods in Efficiency Analysis, New York: Springer Science+Business Media, LLC.

Daraio, C., Simar, L., 2014, Directional distances and their robust versions: Computational and testing issues, European Journal of Operational Research, Vol. 237, pp. 358-369.

Daraio, C., Simar, L., Wilson, P.W., 2020, Fast and efficient computation of directional distance estimators, Annals of Operations Research, Vol. 288, pp. 805-835.

Debreu, G., 1951, The coefficient of resource utilization, Econometrica, Vol. 19, pp. 273-292. 
Deprins, D., Simar, L., Tulkens, H., 1984, Measuring labor-efficiency in post offices, The Performance of Public Enterprises: Concepts and Measurement, Amsterdam: North-Holland, pp. 243-267.

Dibiaggio, L., 2007, Design complexity, vertical disintegration and knowledge organization in the semiconductor industry, Industrial and Corporate Change, Vol. 16, pp. 239-267.

Ernst, D., 2005, Limits to modularity: reflections on recent developments in chip design, Industry and Innovation, Vol. 12, pp. 303-335.

Färe, R.S., Grosskopf, S., Lovell, A.K., 1985, The measurement of efficiency of production, Boston: Kluwer-Nijhoff Publishing.

Farrell, M.J., 1957, The measurement of productive efficiency, Journal of the Royal Statistical Society, Vol. 120, pp. 253-290.

Flamm, K., 2017, Has Moore's law been repealed? An economist's perspective, Computing in Science \& Engineering, Vol. 19, pp. 29-40.

Hall, P., Racine, J.S., Li, Q., 2004, Cross-validation and the estimation of conditional probability densities, Journal of the American Statistical Association, Vol. 99, pp. $1015-1026$.

Hsieh, Y., Lin, N., Chiu, H., 2002, Virtual factory and relationship marketing - a case study of a Taiwan semiconductor manufacturing company, International Journal of Information Management, Vol. 22, pp. 109-126.

Hsu, L.C., 2015, Using a decision-making process to evaluate efficiency and operating performance for listed semiconductor companies, Technological and Economic Development of Economy, Vol. 21, pp. 301-331.

Hwang, H., Choung, J., 2014, The co-evolution of technology and institutions in the catch-up process: the case of the semiconductor industry in Korea and Taiwan, The Journal of Development Studies, Vol. 50, pp. 1240-1260.

Hung, S.W., Lu, W.M., 2008, The comparative productivity efficiency of Taiwan's integrated circuits packaging/testing Firms, Information Systems and Operational Reserach, Vol. 46, pp. 189-198.

Hung, S.W., He, D.S., Lu, W.M., 2014, Evaluating the dynamic performances of business groups from the carry-over perspective: a case study of Taiwan's semiconductor industry, Omega, Vol. 46, pp. 1-10.

Jang, H., Lee, S., Suh, E., 2016, A comparative analysis of the change in R\&D efficiency: a case of R\&D leaders in the technology industry, Technology Analysis \& Strategic Management, Vol. 28, pp. 886-900.

Jeong, S., Park, B.U., Simar, L., 2010, Nonparametric conditional efficiency measures: asymptotic properties, Annals of Operations Research, Vol. 173, pp. 105-122.

Kapoor, R., Adner, R., 2012, What firms make vs. what they know: how firms' production and knowledge boundaries affect competitive advantage in the face of technological change, Organization Science, Vol. 23, pp. 1227-1248.

Kapoor, R., 2013, Persistence of integration in the face of specialization: how firms navigated the winds of disintegration and shaped the architecture of the semiconductor industry, Organization Science, Vol. 24, pp. 1195-1213. 
Koopmans, T.C., 1951, An analysis of production as an efficient combination of activities. In Activity Analysis of Production and Allocation, Ed. T.C. Koopmans. New York: John-Wiley and Sons, Inc.; 33-97. Cowles Commission for Research in Economics, Monograph 13.

Kozmetsky, G., Yue, P., 1998, Comparative performance of global semiconductor companies, Omega, Vol. 26, pp. 153-175.

Kneip, A., Simar, L., Wilson, P.W., 2015, When bias kills the variance: Central limit theorems for DEA and FDH efficiency scores, Econometric Theory, Vol. 31, pp. 394422 .

Kneip, A., Simar, L., Wilson, P.W., 2016, Testing hypotheses in nonparametric models of production, Journal of Business and Economic Statistics, Vol. 34, pp. 435-456.

Kuo, M.H., Yang, C., 2012, Does intellectual capital matter? Assessing the profitability and marketability of IC design companies, Quality \& Quantity, Vol. 46, pp. 1865-1881.

Li, H., He, H., Shan, J., Cai, J., 2019, Innovation efficiency of semiconductor industry in China: a new framework based on generalized three-stage DEA analysis, SocioEconomic Planning Sciences, Vol. 66, pp. 136-148.

Li, Q., Lin, J., Racine, J.S., 2013, Optimal bandwidth selection for nonparametric conditional distribution and quantile functions, Journal of Business \& Economic Statistics, Vol. 31, pp. 57-65.

Li, Y., Huang, M., Chen, D., 2011, Semiconductor industry value chain: characters' technology evolution, Industrial Management \& Data Systems, Vol. 111, pp. 370-390.

Lin, F., Lin, S.W., Lu, W.M., 2019, Dynamic eco-efficiency evaluation of the semiconductor industry: A sustainable development perspective, Environ Monit Assess, Vol. 191, pp. 1-16.

Liu, F., Wang, P., 2008, DEA Malmquist productivity measure: Taiwanese semiconductor companies, International Journal of Production Economics, Vol. 112, pp. 367379 .

Lu, W.M., Hung, S.W., 2010, Assessing the performance of a vertically disintegrated chain by the DEA approach - A case study of taiwanese semiconductor firms, International Journal of Production Research, Vol. 48, pp. 1155-1170.

Lu, W.M., Wang, W.K., Lee, H.L., 2013, The relationship between corporate social responsibility and corporate performance: evidence from the US semiconductor industry, International Journal of Production Research, Vol. 51, pp. 5683-5695.

Macher, J.T., 2006, Technological development and the boundaries of the firm: a knowledge-based examination in semiconductor manufacturing, Management Science, Vol. 52, pp. 826-843.

Macher, J.T., Mowery, D.C., Di Minin, A., 2007, The "non-globalization" of innovation in the semiconductor industry, California Management Review, Vol. 50, pp. 217-242.

Mack, C.A., 2011, Fifty Years of Moore's Law, IEEE Transactions on Semiconductor Manufacturing, Vol. 24, pp. 202-207.

Mastromarco, C., Simar, L. 2015, Effect of FDI and time on catching up: new insights from a conditional nonparametric frontier analysis, Journal of Applied Econometrics Vol. 30, pp. 826-847. 
Monteverde, K., 1995, Technical dialog as an incentive for vertical integration in the semiconductor industry, Management Science Vol. 41, pp. 1624-1638.

Sarma, S., Sun, L., 2017, The genesis of fabless business model: institutional entrepreneurs in an adaptive ecosystem, Asia Pacific Journal of Management, Vol. 34, pp. 587-617.

Shin, N., Kraemer, K., Dedrick, J., 2017, R\&D and firm performance in the semiconductor industry, Industry and Innovation, Vol. 24, pp. 280-297.

Simar, L., Vanhems, A., 2012 Probabilistic characterization of directional distances and their robust versions, Journal of Econometrics, Vol. 166, pp. 342-354.

Simar, L., Wilson, P.W., 2007, Estimation and inference in two-stage, semi-parametric models of productive efficiency, Journal of Econometrics, Vol. 136, pp. 31-64.

Simar, L., Wilson, P.W., 2011, Two-stage DEA: Caveat emptor, Journal Productivity Analysis, Vol. 36, pp. 205-218.

Simar, L., Wilson, P.W., 2015, Statistical approaches for non-parametric frontier models: a guided tour, International Statistical Review, Vol. 83, pp. 77-110.

Simar, L., Wilson, P.W., 2020, Hypothesis testing in nonparametric models of production using multiple sample splits, Journal Productivity Analysis, Vol. 53, pp. 287-303.

Tan, H., Mathews, J.A., 2010, Cyclical industrial dynamics: the case of the global semiconductor industry, Technological Forecasting \& Social Change, Vol. 77, pp. 344353.

Toma, P., 2020, Size and productivity: a conditional approach for Italian pharmaceutical sector, Journal Productivity Analysis, Vol. 54, pp. 1-12.

Tsai, C.H., Wu, H.Y., Chen, I.S., Chen, J.K., Ye, R.W., 2017, Exploring benchmark corporations in the semiconductor industry based on efficiency, Journal of High Technology Management Research, Vol. 28, pp. 188-207.

Wilson, P.W., 2008, FEAR 1.0: a software package for frontier efficiency analysis with R, Socio-Economic Planning Sciences, Vol. 42, pp. 247-254.

Wilson, P.W., 2018, Dimension reduction in nonparametric models of production, European Journal of Operational Research, Vol. 267, pp. 349-367.

Wu, W.Y., Tsai, H.J., Cheng, K.Y., Lai, M., 2006, Assessment of intellectual capital management in Taiwanese IC design companies: using DEA and the Malmquist productivity index, R\&D Management, Vol. 36, pp. 531-545. 
Table 1: Summary Statistics for 1999-2018 Pooled Data

\begin{tabular}{ccccccc}
\hline \hline Variable & Min & Q1 & Median & Mean & Q3 & Max \\
\hline$X_{1}$ & 0.001 & 0.160 & 0.486 & 3.082 & 2.011 & 107.600 \\
$X_{2}$ & 0.001 & 24.170 & 88.008 & 475.252 & 301.645 & $18,226.000$ \\
$X_{3}$ & 0.000 & 4.302 & 18.330 & 160.217 & 67.001 & $13,543.000$ \\
$X_{4}$ & 0.549 & 5.885 & 20.087 & 125.406 & 67.185 & $1,982.015$ \\
$X_{f}$ & 0.005 & 6.065 & 27.787 & 554.060 & 174.405 & $48,976.000$ \\
$Y_{1}$ & 0.003 & 47.283 & 161.799 & 1064.110 & 563.655 & $70,848.000$ \\
$Y_{2}$ & 0.175 & 44.279 & 151.749 & 1114.748 & 487.730 & $74,563.000$ \\
\hline $\begin{array}{c}\text { Obs. } \\
\text { Uniq. Obs. }\end{array}$ & & & & 5,136 & & \\
\hline \hline
\end{tabular}

NOTE. The unit of $X_{1}$ is thousand employees.

The units of the variables except $X_{1}$ are US\$ million.

All values have been adjusted to 2018 US $\$$ by GDP deflator.

Obs. denotes the total number of observations in 1999-2018.

Uniq. Obs. denotes the unique number of companies in 1999-2018. 
Table 2: Number of Observations By Business Model

\begin{tabular}{|c|c|c|c|c|c|}
\hline \multirow[b]{2}{*}{ Year } & \multirow[b]{2}{*}{ All } & \multicolumn{3}{|c|}{ — Number of Companies } & \multirow[b]{2}{*}{ Fabless } \\
\hline & & IDM & Foundry & $\mathrm{A} \& \mathrm{~T}$ & \\
\hline 1999 & 125 & 38 & 10 & 9 & 68 \\
\hline 2000 & 149 & 43 & 10 & 15 & 81 \\
\hline 2001 & 155 & 46 & 10 & 16 & 83 \\
\hline 2002 & 213 & 48 & 17 & 27 & 121 \\
\hline 2003 & 241 & 49 & 19 & 30 & 143 \\
\hline 2004 & 264 & 54 & 21 & 30 & 159 \\
\hline 2005 & 260 & 54 & 17 & 27 & 162 \\
\hline 2006 & 267 & 56 & 20 & 30 & 161 \\
\hline 2007 & 269 & 52 & 21 & 33 & 163 \\
\hline 2008 & 278 & 51 & 20 & 35 & 172 \\
\hline 2009 & 290 & 53 & 21 & 36 & 180 \\
\hline 2010 & 300 & 59 & 23 & 38 & 180 \\
\hline 2011 & 298 & 60 & 22 & 39 & 177 \\
\hline 2012 & 301 & 61 & 22 & 38 & 180 \\
\hline 2013 & 313 & 65 & 24 & 41 & 183 \\
\hline 2014 & 302 & 62 & 25 & 43 & 172 \\
\hline 2015 & 288 & 59 & 24 & 42 & 163 \\
\hline 2016 & 283 & 54 & 23 & 44 & 162 \\
\hline 2017 & 275 & 51 & 23 & 45 & 156 \\
\hline 2018 & 265 & 48 & 22 & 44 & 151 \\
\hline Obs. & 5,136 & 1,063 & 394 & 662 & 3,017 \\
\hline Uniq. Obs. & 470 & 83 & 36 & 63 & 288 \\
\hline
\end{tabular}

NOTE. Obs. denotes the total number of observations in 1999-2018.

Uniq. Obs. denotes the unique number of companies in 1999-2018. 
Table 3: Results of Convexity Test (Hyperbolic-Orientation)

\begin{tabular}{llll}
\hline \hline Year & $\mathrm{N}$ & Statistic & $p$-value \\
\hline 1999 & 125 & 2.222 & 0.011 \\
2000 & 149 & 1.594 & 0.006 \\
2001 & 155 & 1.853 & 0.040 \\
2002 & 213 & 3.138 & 0.005 \\
2003 & 241 & 2.901 & 0.001 \\
2004 & 264 & 3.440 & 0.000 \\
2005 & 260 & 3.238 & 0.000 \\
2006 & 267 & 3.651 & 0.000 \\
2007 & 269 & 3.915 & 0.003 \\
2008 & 278 & 3.227 & 0.008 \\
2009 & 290 & 2.162 & 0.057 \\
2010 & 300 & 2.890 & 0.006 \\
2011 & 298 & 2.102 & 0.088 \\
2012 & 301 & 1.014 & 0.174 \\
2013 & 313 & 1.989 & 0.020 \\
2014 & 302 & 3.552 & 0.001 \\
2015 & 288 & 1.452 & 0.041 \\
2016 & 283 & 2.053 & 0.018 \\
2017 & 275 & 4.831 & 0.000 \\
2018 & 265 & 4.963 & 0.000 \\
\hline
\end{tabular}

NOTE. I use 100 splits and 1000 bootstrap replications. 
Table 4: Test of Separability conditional on $Z_{1}$ and $Z_{2}$ (with Dimension Reduction, $p$ $=2, q=1$, and directional distance measure)

\begin{tabular}{|c|c|c|c|c|}
\hline & \multicolumn{2}{|c|}{$-\tau_{1}-$} & \multicolumn{2}{|c|}{$-\tau_{2}-$} \\
\hline & Statistic & $p$-value & Statistic & $p$-value \\
\hline & - & \multicolumn{2}{|c|}{ Conditional on $Z_{1}$} & - \\
\hline Fabless VS. IDM & 7.567 & 0.000 & 0.992 & 0.925 \\
\hline Fabless VS. OSAT & 4.126 & 0.000 & 1.000 & 0.000 \\
\hline IDM VS. OSAT & 3.883 & 0.000 & 0.870 & 0.715 \\
\hline \multirow{3}{*}{ Pooled VS. Optimal Time } & - & \multicolumn{2}{|c|}{ Conditional on $Z_{2}$} & \\
\hline & 3.230 & 0.000 & 0.983 & 0.000 \\
\hline & - & \multicolumn{2}{|c|}{ Conditional on $Z_{1}, Z_{2}$} & - \\
\hline 2-Year Groups & 26.273 & 0.000 & 1.000 & 0.000 \\
\hline 4-Year Groups & 18.572 & 0.000 & 1.000 & 0.000 \\
\hline 5-Year Groups & 17.242 & 0.000 & 1.000 & 0.000 \\
\hline Optimal Time & 19.891 & 0.000 & 1.000 & 0.000 \\
\hline
\end{tabular}


Table 5: Summary statistics of the efficiency scores by directional distance estimator

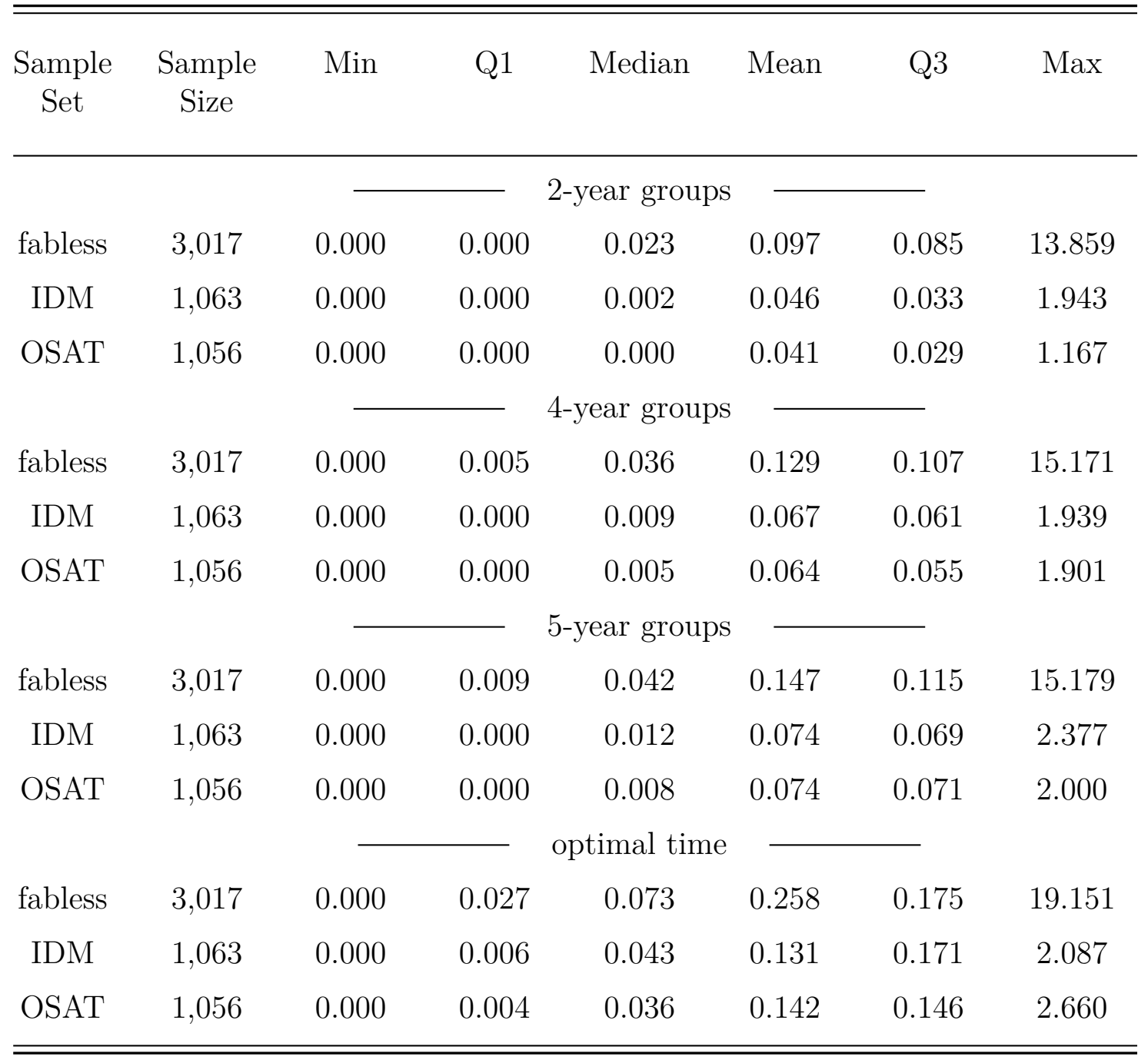



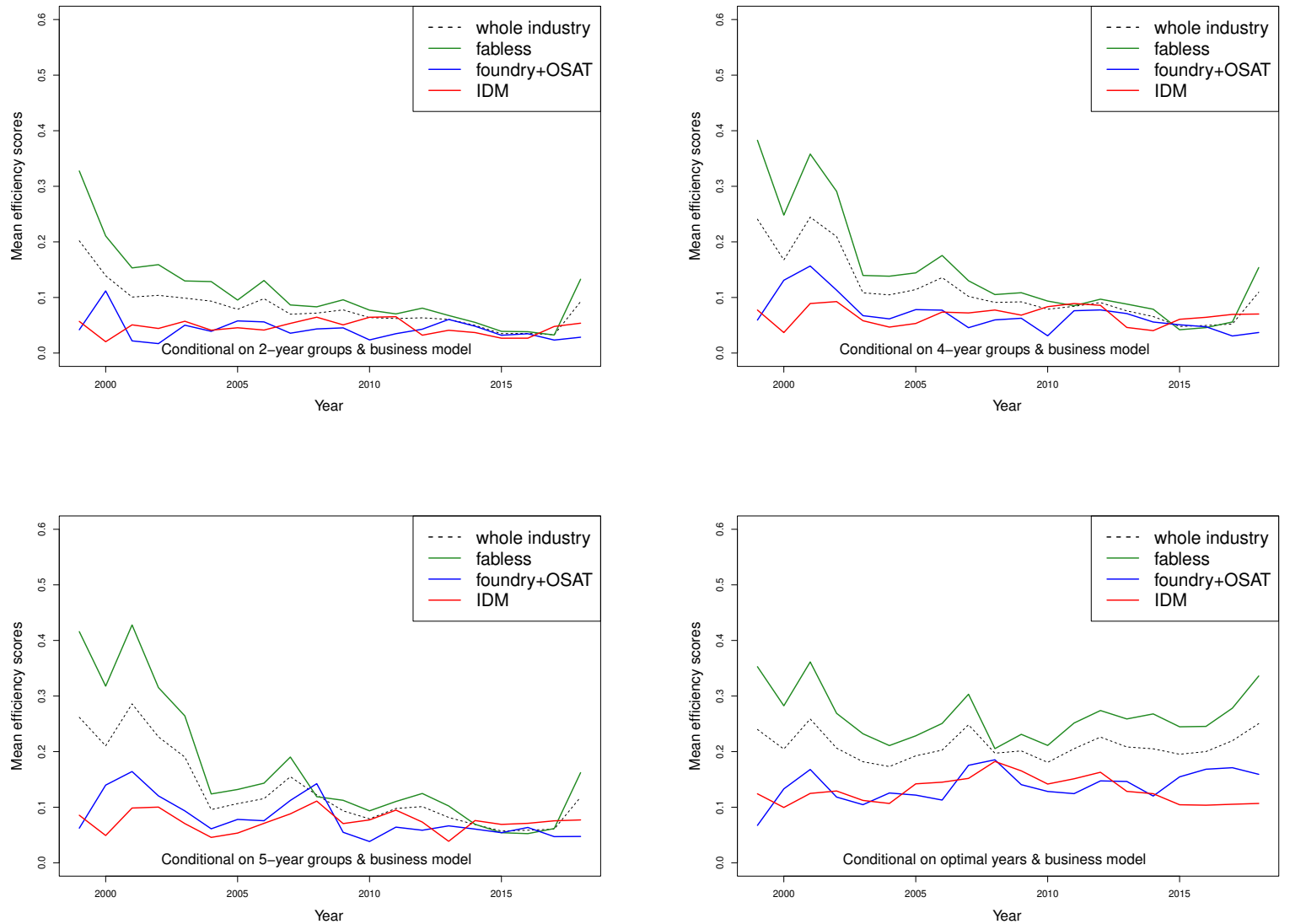

Figure 1: Mean $\beta$ conditional on both year and business model 


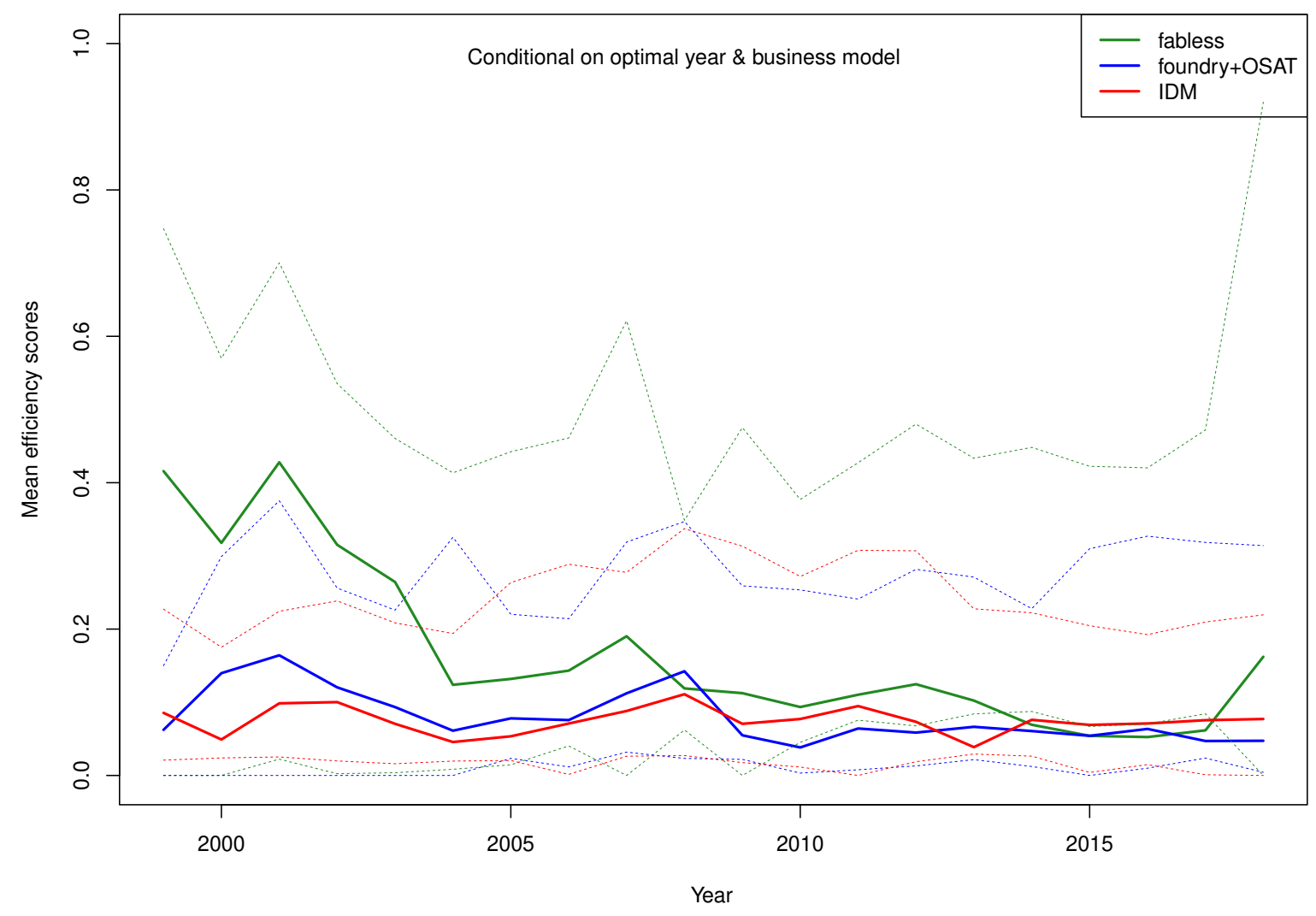

Figure 2: Mean efficiency conditional on optimal year and business model with $95 \%$ confidence interval 

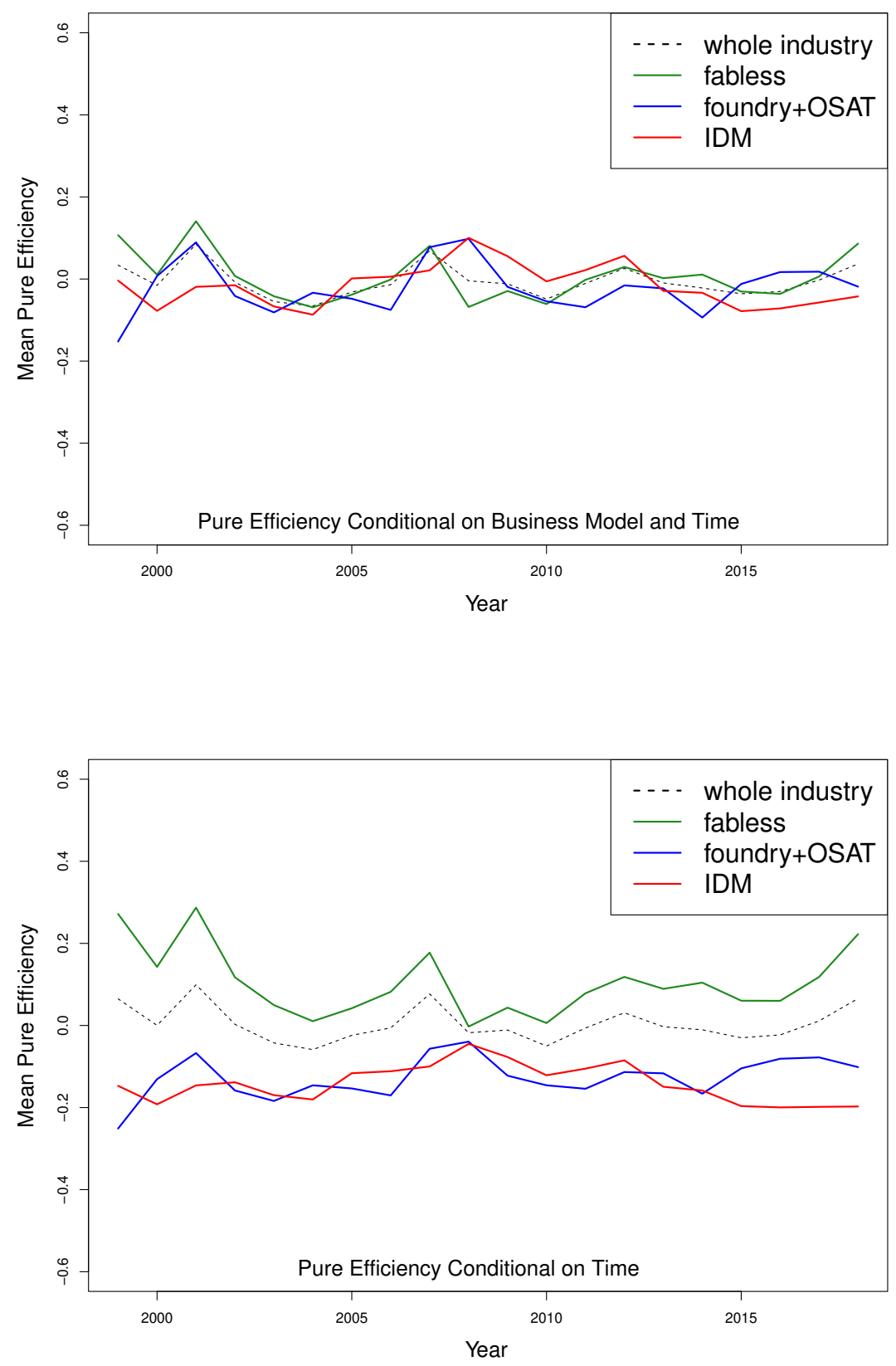

Figure 3: Pure efficiency 